Psychotherapeut $2021 \cdot 66: 524-532$ https://doi.org/10.1007/s00278-021-00528-z Angenommen: 8. Juli 2021 Online publiziert: 1. September 2021 ○ Der/die Autor(en) 2021

\section{Entwicklung von Affiliation während der Psychotherapieausbildung}

\author{
Auf dem Weg zu einer wohlwollenden Haltung
}

Zusammenfassung

Fragestellung: Die Studie untersuchte die Entwicklung von Affiliation bei psychotherapeutischen Ausbildungskandidat:innen in Bezug auf ihr therapeutisches Handeln, ihre Selbstwahrnehmung und ihre Wahrnehmung von Klient:innen. Zudem wurde der Einfluss von Bindungsrepräsentationen und Selbsterfahrung auf die Affiliation betrachtet.

Material und Methode: In einem naturalistischen Prä-Post-Design bewerteten Ausbildungskandidat:innen unterschiedlicher therapeutischer Orientierungen ( $n=126)$ verschiedene Affiliationsdimensionen in schwierigen Therapiesituationen (Intrex Questionnaire Short Form) über den Abstand von 3 Jahren. Bindungsangst und Bindungsvermeidung wurden zu Beginn (Experiences in Close Relationships, ECRRD), Länge und Zufriedenheit der Selbsterfahrung am Ende des Erhebungszeitraums erfasst. Die Zusammenhangsanalysen wurden in Mehrebenenmodellen realisiert. Ergebnisse: Die Affiliation im eigenen therapeutischen Verhalten und in der Wahrnehmung des Klient:innenverhaltens zeigte Steigerungen kleiner Effektgröße, wobei Bindungsvermeidung eine geringere Affiliation im eigenen Verhalten voraussagte. Die selbstbezogene Affiliation nahm mit einer mittleren Effektgröße $\mathrm{ab}$; hierbei war Bindungsangst mit einer niedrigeren Affiliation assoziiert. Die Selbsterfahrungsdauer wies einen negativen Zusammenhang mit der Wahrnehmung des Klient:innenverhaltens auf; dieser Effekt kehrte sich bei hoher Bindungsvermeidung um. Die Zufriedenheit mit Selbsterfahrung hatte keinen Einfluss auf die Affiliationsentwicklung.

Schlussfolgerung: Die interpersonelle und intrapsychische Affiliation von angehenden Psychotherapeut:innen ist z. T. durch Bindungsrepräsentationen geprägt, unterliegt aber Entwicklungsprozessen. Die Abnahme selbstbezogener Affiliation zeigt die Notwendigkeit für kompensatorische und ressourcenstärkende Maßnahmen in der Psychotherapieausbildung.

\title{
Schlüsselwörter
}

Bindungsrepräsentation · Interpersonelle Wahrnehmung · Selbstwahrnehmung · Selbsterfahrung · Therapeuteneffekte

Vergleicht man das therapeutische Handeln sowie dazugehörige Gedanken und Gefühle von sehr effektiven und wenig effektiven Psychotherapeut:innen, unterscheiden sich diese u.a. hinsichtlich einer wohlwollenden Haltung ihren Kli- ent:innen sowie sich selbst gegenüber. Mit Blick auf die Reform der Psychotherapieausbildung in Deutschland stellen sich daher die Fragen, ob es sich dabei um ein individuelles Merkmal oder eine formbare Eigenschaft handelt, und 
welche Faktoren zu einer potenziellen Entwicklung bei Ausbildungskandidat:innen beitragen. Die vorliegende Längsschnittstudie untersucht diese Thematik in einem naturalistischen Setting.

\section{Hintergrund}

\section{Merkmale effektiver Therapeut:innen}

Der Begriff der Therapeuteneffekte beschreibt den robusten Forschungsbefund, dass sich Psychotherapeut:innen in ihrer Effektivität aufgrund interindividueller Merkmale unterscheiden. In einer aktuellen Metaanalyse gehen Johns et al. (2019) davon aus, dass $5 \%$ der Ergebnisvarianz in naturalistischen Therapieverläufen und $8 \%$ in randomisierten klinischen Studien durch Therapeuteneffekte erklärt werden. Psychotherapeut:innen mit überdurchschnittlich guten Therapieergebnissen zeichnen sich durch ihre interpersonellen Fähigkeiten aus: So trägt ein therapeutisches Handeln, geprägt von Wärme, positiver Wertschätzung, klarer positiver Kommunikation und Kritikfähigkeit, zur Entwicklung eines tragfähigen therapeutischen Arbeitsbündnisses bei (Nienhuis et al. 2018) und sagt bessere TherapieOutcomes voraus (Heinonen und NissenLie 2020).

Diesen zuträglichen interpersonellen Eigenschaften von Psychotherapeut:innen ist gemeinsam, dass sie von einer wohlwollenden, zugewandten Grundhaltung geprägt sind, die sich positiv auf den Therapieprozess auswirken kann (Bruck et al. 2006; Heinonen und Nissen-Lie 2020; Henry et al. 1990; Schut et al. 2005). Theoretisch lässt sich dies in der Dimension der Affiliation der Structural Analysis of Social Behavior (SASB; Benjamin 1974) operationalisieren. Entsprechend dem SASB-Modell kann interpersonelles Verhalten anhand der beiden Dimensionen der Affiliation (z.B. liebend vs. hassend) und der Interdependenz (z.B. kontrollierend vs. freilassend) klassifiziert werden. Zudem unterscheidet es drei Betrachtungsperspektiven, wovon zwei das tatsächliche interpersonelle Verhalten beschreiben (transitiv-agieren und intransitiv-reagieren), während eine in- trapsychische Perspektive beschreibt, wie ein Individuum sich selbst während der Interaktion mit anderen wahrnimmt. Aus der interpersonellen Perspektive ist affiliatives Verhalten in naturalistischen Psychotherapiestudien mit positiven Veränderungsprozessen und Ergebnissen assoziiert, während ein eher ablehnendes oder feindseliges therapeutisches Verhalten mit schlechteren Therapieergebnissen verbunden ist (Henry et al. 1990; Schut et al. 2005). Auf der intrapsychischen Ebene hängen höhere Affiliationswerte, also eine stärker selbstliebende Haltung, mit mehr therapeutischer Selbstwirksamkeit (Taubner et al. 2013), besseren Outcomes sowie gleichmäßigeren und tiefgründigeren Therapiesitzungen zusammen (Bruck et al. 2006).

\section{Bindungsrepräsentationen und Selbsterfahrung}

In Anbetracht der Bedeutung von Affiliation für Therapieprozesse und vor dem Hintergrund der aktuellen Forschung zur Kompetenzentwicklung in der Psychotherapieausbildung stellt sich die Frage, ob es sich bei interpersoneller und intrapsychischer Affiliation, um ein stabiles "Trait" oder eine formbare Eigenschaft handelt. In bisherigen Therapiestudien wurde die intrapsychische Affiliation meist als stabile Eigenschaft von Psychotherapeut:innen behandelt (Bruck et al. 2006; Henry et al. 1990; Nissen-Lie et al. 2017). Allerdings fanden Taubner et al. (2013) in einer Studie mit 171 Ausbildungskandidat:innen (AK) eine Zunahme von intrapsychischer Affiliation über 3 Jahre Therapieausbildung, was für die Annahme einer formbaren Eigenschaft spricht. Zur Entwicklung der interpersonellen Affiliation während der Ausbildung liegen bisher noch keine Befunde vor.

Im Sinne eines Trait geht der theoretische Rahmen des SASB davon aus, dass aktuelle Kommunikations- und Verhaltensmuster frühkindliche interpersonelle Erfahrungen widerspiegeln (Benjamin 1974). Entsprechend wird die inter- und intrapersonelle Affiliation in der theoretischen Literatur mit Bindungsrepräsentationen in $\mathrm{Zu}$ sammenhang gebracht (Gallo et al. 2003; Pincus et al. 1999). Nach Annahme der Bindungstheorie entwickeln Kinder in Interaktionen mit frühen Bezugspersonen in- ternalisierte Arbeitsmodelle, verinnerlichte Schemata des Selbst und der Anderen (Bowlby 1988). Diese Bindungsrepräsentationen prägen die Emotionsregulation, Erinnerung sowie soziale Informationsverarbeitung eines Individuums (Dykas und Cassidy 2011) und können auf diese Weise zukünftige interpersonelle Interaktionen beeinflussen (Sroufe et al. 2005). So nehmen Individuen mit einer sicheren Bindungsrepräsentation sich selbst als liebenswert und andere als verfügbar und vertrauenswürdig wahr und haben damit eine höhere Wahrscheinlichkeit affiliative Beziehungen zu entwickeln. Der Grad der Bindungssicherheit kann anhand von zwei Dimensionen genauer differenziert werden: Bindungsvermeidung zeigt das Ausmaß von Unbehagen mit interpersoneller Nähe, während Bindungsangst die Ausprägung von Ängsten vor Zurückweisung oder Verlassenwerden anzeigt (Mikulincer und Shaver 2007; Shaver et al. 1996).

Bindungsrepräsentationen wurden sowohl in der theoretischen Literatur (Slade und Holmes 2019) als auch empirisch (Mohr et al. 2005; Rubino et al. 2000) mit affiliativem Erleben und Verhalten im Klient:innenkontakt in Verbindung gebracht. Dabei könnten schwierige Therapiesituationen vermehrt zu interpersonellem Stress führen. Dieser Stress könnte bindungsbezogene Repräsentationen der Psychotherapeut:innen aktivieren (Mikulincer et al. 2002) und auf diese Weise das Ausmaß an Affiliation in ihrem situativen Verhalten und Wahrnehmen prägen. So berichten Schauenburg et al. (2010), dass sichere Bindungsrepräsentationen von Psychotherapeut:innen nur bei interpersonell schwierigen Klient:innen mit einer besseren therapeutischen Allianz assoziiert waren. Darüber hinaus werden hohe Bindungsvermeidung von Psychotherapeut:innen mit vermehrt feindseligen Gegenübertragungen (Mohr et al. 2005) und hohe Bindungsangst mit weniger empathischen Reaktionen (Rubino et al. 2000) und einer geringeren therapeutischen Selbstwirksamkeit (Deal et al. 2011) in Verbindung gebracht. Der Einfluss von Bindungsrepräsentationen auf die interpersonelle und intrapsychische Affiliation von AK wurde bisher jedoch nicht direkt untersucht. 
Während der Therapieausbildung könnten Selbsterfahrung oder Lehrtherapien zu einer Förderung von Affiliation im Sinne einer formbaren Eigenschaft beitragen. Sie bieten einen Raum, sich mit eigenen interpersonellen Mustern auseinanderzusetzen, emotionale Resilienz und Empathie zu entwickeln sowie zwischen Anteilen der AK und ihrer Klient:innen zu differenzieren (Murphy et al. 2018). Auf diese Weise könnten AK negative Emotionen gegenüber ihren Klient:innen bearbeiten sowie Selbstzweifel überwinden und dadurch mehr interpersonelle und intrapsychische Affiliation entwickeln. Entsprechend dieser Annahme zeigten Psychotherapeut:innen mit Selbsterfahrung vergleichsweise bessere therapeutische Allianzen und ein höheres Vertrauen in eigenes therapeutisches Handeln (Gold und Hilsenroth 2009). In ihrer Studie mit 171 AK fanden Taubner et al. (2013), dass therapeutische Selbstwirksamkeit durch eine Interaktion aus der Zufriedenheit mit Selbsterfahrung und Veränderungen der intrapsychischen Affiliation vorausgesagt wurde. Es ist bisher jedoch nicht bekannt, ob Selbsterfahrung einen direkten Einfluss auf die Entwicklung von interpersoneller und intrapsychischer Affiliation hat.

\section{Naturalistische Längsschnittstudie}

\section{Fragestellung}

In der vorliegenden Studie wird die Entwicklung der interpersonellen und intrapsychischen Affiliation von 184 psychotherapeutischen AK über den Verlauf von 3 Jahren erhoben. Als Indikator für intra- und interpersonell herausfordernde Situationen wird die Einschätzung der Affiliation in schwierigen Zeiten erfasst. Als mögliche Einflussvariablen werden in dieser Studie die dimensionalen Einschätzungen von Bindungsangst und Bindungsvermeidung sowie der Umfang von und die Zufriedenheit mit der Selbsterfahrung in der Psychotherapieausbildung untersucht. Entsprechend der bisherigen Literatur wird Folgendes erwartet:

- eine Steigerung aller Affiliationsbereiche über den 3-jährigen Ausbildungsverlauf;

- ein negativer Zusammenhang zwischen dem Ausmaß von Bindungs- angst und Bindungsvermeidung mit den Affiliationswerten der AK. Mehr Bindungsangst und Bindungsvermeidung zu Beginn der Erhebung führen zu einer niedrigeren interpersonellen und intrapsychischen Affiliation nach 3 Jahren Ausbildung.

Als explorative Fragestellung wird untersucht, welchen Einfluss die Länge von und Zufriedenheit mit Selbsterfahrung während der Ausbildung auf Affiliationswerte nach 3 Jahren haben.

\section{Material und Methode}

\section{Studiendesign}

Bei der vorliegenden Studie handelt es sich um eine Sekundäranalyse von Daten, die im Rahmen der Studie Kompetenzentwicklung von PsychotherapeutInnen in Ausbildung (KPA; Taubner et al. 2015; DFG-Fördernummern MO 2008/2-1, MO 2008/2-2) erhoben wurden. Diese Längsschnittstudie untersuchte die Entwicklung von fachlich-konzeptuellen, personalen und Beziehungskompetenzen angehender psychologischer Psychotherapeut:innen in einem naturalistischen Prä-Post-Design. Die Datenerhebung der Prämessung (T1) fand in den Jahren 2011 und 2012 statt, die Postmessung (T2) 3 Jahre später.

\section{Rekrutierung und Stichprobe}

Im Vorfeld der Studie wurden 29 verschiedene Ausbildungsinstitute innerhalb der Bundesrepublik Deutschland kontaktiert, die eine postgraduale Psychotherapieausbildung in einem der 3 zu diesem Zeitpunkt sozialrechtlich anerkannten Verfahren (kognitive Verhaltenstherapie, tiefenpsychologisch fundierte Psychotherapie, analytische Psychotherapie) anboten. Dabei wurde eine Gleichverteilung der therapeutischen Orientierungen sowie der regionalen Lage angestrebt. Insgesamt stimmten 17 Ausbildungsinstitute (59\%) einer Studienkooperation zu. Als Grund für eine Nichtteilnahme wurden hohe Arbeitsbelastungen während der Ausbildung angegeben. Darüber hinaus konnten keine weiteren Informationen über Gründe der Nichtteilnahme gesammelt werden.
Insgesamt nahmen 184 AK an der ersten Erhebung teil. Mit 47,3\% $(n=87)$ waren die Teilnehmenden der tiefenpsychologisch fundierten Therapieausbildung am häufigsten vertreten, gefolgt von den Teilnehmenden der Verhaltenstherapieausbildung (34,7\%, $n=64)$, während die Teilnehmenden der analytischen Ausbildung die kleinste Gruppe ausmachten $(18 \%, n=33)$. Die Teilnehmenden wiesen ein mittleres Alter von 31,42 Jahren (SD $\pm 6,67$ Jahre, Range 24 bis 55 Jahre) auf, befanden sich im Mittel im 2,3 Semester ( $S D \pm 1,82$ Semester, Range ein bis 12 Semester) und waren zu 84,2\% weiblich. Alle Teilnehmenden gaben als berufsqualifizierenden Abschluss ein Psychologiestudium an, $8,7 \%$ verfügten zusätzlich über einen weiteren akademischen Abschluss (davon $44 \%$ Geisteswissenschaft, $31 \%$ Sozialwissenschaften und $25 \%$ andere Fachrichtungen). An der Post-Erhebung nahmen 126 AK teil (Drop-out-Rate: $32 \%$ ). Um Veränderungen der Affiliation über die Zeit verlässlich bestimmen zu können, bildet diese Teilstichprobe die Datengrundlage der vorliegenden Studie.

\section{Maße}

Affiliation. Der Intrex Questionnaire Short Form (Intrex; Benjamin 1995) wurde angewendet, um die wahrgenommene interpersonelle und intrapsychische Affiliation über den Verlauf der Therapieausbildung zu messen. Dieses Selbstberichtmaß basiert auf dem SASB-ClusterModell (Benjamin 1974), das 3 mögliche Betrachtungsfoci zwischenmenschlichen Handelns konzeptualisiert (interpersonell: transitiv, intransitiv; intrapsychisch). In allen drei Foci wird Verhalten anhand seiner Ausprägung auf den beiden zugrunde liegenden Dimensionen der Affiliation und der Interdependenz klassifiziert und einem von 8 Clustern zugeordnet.

Die Teilnehmenden wurden aufgefordert, ihr selbstbezogenes Verhalten (Beispielitem: „Ich nehme mich so, wie ich bin, mit all meinen Stärken und Schwächen“), ihr eigenes interpersonelles Verhalten gegenüber Klient:innen (Beispielitem: „Ich gebe ihr:ihm sehr freundlich Anleitung, Schutz und Fürsorge“) sowie das Verhalten ihrer Klient:innen ihnen gegenüber (Beispielitem mit geringer Affiliation „Ohne einen Gedanken an die Folgen, greift 
Tab. 1 Deskriptivstatistiken der untersuchten Variablen

\begin{tabular}{|c|c|c|c|c|}
\hline & $M$ & \pm SD & Minimum & Maximum \\
\hline \multicolumn{5}{|l|}{ SASB Intrex Affiliation } \\
\hline \multicolumn{5}{|c|}{ Selbstbezogene Affiliation } \\
\hline T1 & 23,88 & $\pm 39,43$ & $-70,50$ & 96,30 \\
\hline T2 & 3,53 & $\pm 44,94$ & $-100,80$ & 100,80 \\
\hline \multicolumn{5}{|c|}{ Therapeutische Affiliation } \\
\hline T1 & 26,68 & $\pm 32,65$ & $-82,80$ & 89,10 \\
\hline $\mathrm{T} 2$ & 34,66 & $\pm 24,93$ & $-27,90$ & 96,90 \\
\hline \multicolumn{5}{|l|}{ Klient:innenaffiliation } \\
\hline T1 & $-20,76$ & $\pm 35,49$ & $-96,90$ & 94,05 \\
\hline $\mathrm{T} 2$ & $-13,77$ & $\pm 32,87$ & $-96,30$ & 77,25 \\
\hline \multicolumn{5}{|l|}{ Prädiktoren } \\
\hline \multicolumn{5}{|l|}{$E C R$} \\
\hline Bindungsangst & 2,53 & $\pm 0,99$ & 1,00 & 5,11 \\
\hline Bindungsvermeidung & 2,19 & $\pm 0,79$ & 1,00 & 6,11 \\
\hline \multicolumn{5}{|l|}{ Selbsterfahrung } \\
\hline Stunden & 153,63 & $\pm 159,89$ & 7 & 1600 \\
\hline Zufriedenheit & 3,13 & $\pm 0,89$ & 0 & 4 \\
\hline \multicolumn{5}{|l|}{ Kovariaten } \\
\hline Alter & 31,46 & $\pm 6,52$ & 24 & 55 \\
\hline Semester & 2,24 & $\pm 1,74$ & 1 & 12 \\
\hline
\end{tabular}

sie:er mich voller Ablehnung und Zerstörungswut heftig $\mathrm{an}^{\prime \prime}$ ) im therapeutischen Kontext zum schlechtesten Zeitpunkt zu beurteilen. Die originale Itemskalierung (0-100) wurde auf einen Wertebereich von 0-6 modifiziert. Anschließend wurden die einzelnen Clusterwerte zu einem generellen Affiliationsvektor aggregiert (Pincus et al. 1998) und die Affiliationswerte des transitiven und intransitiven Fokus jeweils personenspezifisch aggregiert (Pincus et al. 1999). Daraus ergaben sich die 3 Skalen Affiliation des eigenen therapeutischen Handelns, Affiliation des Verhaltens der Klient:innen und Affiliation der Ausbildungskandidat:innen in Bezug auf sich selbst. Diese wiesen in der vorliegenden Stichprobe akzeptable bis gute interne Konsistenzen auf $(a=0,77-0,81)$.

Bindungsrepräsentationen. Die deutschsprachige Version des Fragebogens Experiences in Close Relationships Revised (ECR-RD; Ehrenthal et al. 2009) wurde genutzt, um Bindungsrepräsentationen zu erfassen. Es handelt sich um ein Selbstberichtinstrument mit 36 Items, das Bindungsstrategien Erwachsener in engen Beziehungen anhand der beiden Dimensionen Bindungsangst (Beispielitem: „Ich mache mir häufig Sorgen um meine Beziehungen") und Bindungsvermeidung (Beispielitem: „Mir wird unwohl, wenn mein:e Partner:in mir sehr nahe sein will“) erfasst. Geringe Werte auf beiden Dimensionen weisen auf eine sichere Bindungsrepräsentation hin. Das Instrument verfügt über gute Konstruktvalidität (Ehrenthal et al. 2009) und wies in der vorliegenden Stichprobe gute interne Konsistenzen auf (Bindungsangst: $a=0,93$; Bindungsvermeidung: $a=0,90$ ). apeutic Attitudes Ausbildungsversion (Sandell et al. 2008) wurde eingesetzt, um persönliche und ausbildungsbezogene Merkmale zu erfassen. In der vorliegenden Studie wurden daraus lediglich quantitative Angaben zur Selbsterfahrung übernommen. In den Analysen wurde die kumulierte Gesamtzahl jeglicher Selbsterfahrungsstunden zu T2 als Indikator für die Selbsterfahrungslänge herangezogen, nachdem Voranalysen keinen signifikan-
Selbsterfahrung. Der Fragebogen Ther- ten Einfluss von Selbsterfahrung vor der Therapieausbildung auf Affiliationsmaße zu T1 fanden. Die Zufriedenheit der Teilnehmenden mit ihrer ausbildungsbezogenen Selbsterfahrung wurde mit einem Item auf einer 5-Punkte-LikertSkala von 0 („,überhaupt nicht zufrieden“) bis 4 (,sehr zufrieden“) erfasst.

\section{Statistische Analysen}

Bei der Datenauswertung wurden in Anbetracht des Drop-outs nur Teilnehmende mit vollständigen Affiliationswerten zu T1 und T2 berücksichtigt (Jakobsen et al. 2017). Dieser Datensatz enthielt 3,1\% fehlende Werte. Sequenzielle t-Tests zwischen den Teilnehmenden ergaben keine Hinweise auf systematische Muster fehlender Werte. Daher wurden fehlende Daten als „missing at random" eingestuft und per multipler Imputation mit dem R Package MICE imputiert. Vier Teilnehmende wiesen univariate Ausreißerwerte auf, die als kritisch einzustufen sind ( $z> \pm 3,29$; Tabachnick und Fidell 2014) und wurden daher aus den Analysen ausgeschlossen.

Um die Affiliationswerte der 3 unterschiedlichen Wahrnehmungsperspektiven zu T2 vorauszusagen, wurden 3 Multilevelmodelle berechnet. Dabei wurden zunächst Baseline-Modelle ohne Prädiktoren aufgestellt, die die Affiliationswerte beider Messzeitpunkte über die Individuen verteilten. Das Vorliegen nennenswerter Intraklassenkorrelationen in solchen Modellen weist auf interindividuelle Unterschiede hin. Anschließend wurden die Zeit als Prädiktor auf Level 1 sowie Bindungsrepräsentationen und Selbsterfahrungsmaße als Prädiktoren auf Level 2 (alle als "fixed effect") spezifiziert. Zudem wurden Alter, Geschlecht, therapeutisches Verfahren und Ausbildungssemester als Kovariaten in die Modelle aufgenommen. Eine visuelle Überprüfung von Residual- und QQPlots ergab keine Hinweise auf signifikante Nonnormalität oder Heteroskedastizität.

Die beiden Bindungsmaße zeigten im Mittel keine signifikanten Veränderungen über den Verlauf der Therapieausbildung (Bindungsangst: $p=0,69$; Bindungsvermeidung: $p=0,64)$, daher wurden die Werte zu Beginn der Therapieausbildungen als Indikatoren eines stabilen Persönlichkeit-Trait konzeptualisiert. 

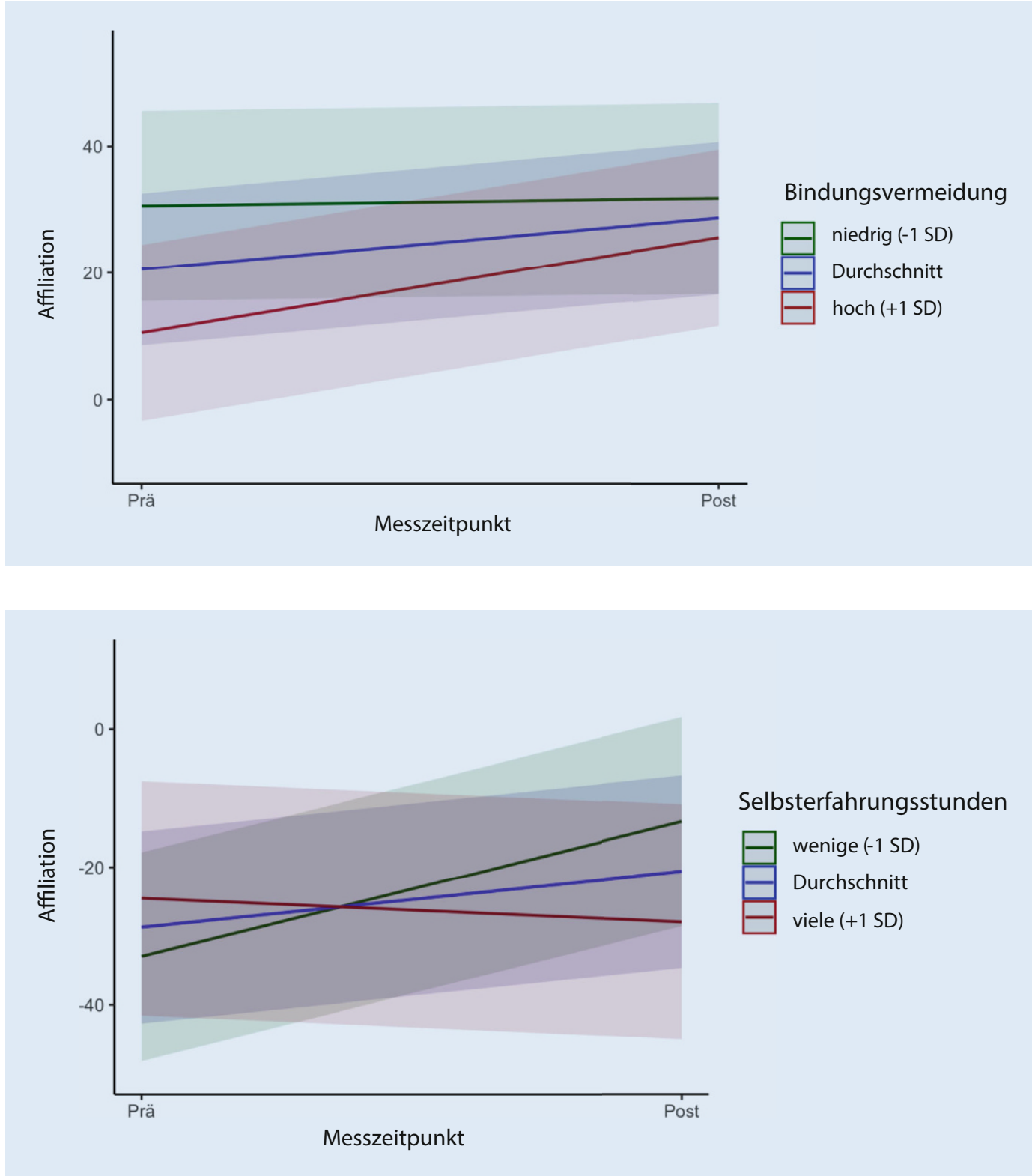

Abb. 1 - Entwicklung der Affiliation im therapeutischen Verhalten in Abhängigkeit von Bindungsvermeidung
Abb. $2<$ Interaktionseffekt von Selbsterfahrungsstunden und Zeit auf wahrgenommene Affiliation im Verhalten von Klient:innen

\section{Ergebnisse}

Die Deskriptivstatistiken aller Prädiktoren, Kriteriumsvariablen und Kovariaten sind in - Tab. 1 aufgeführt.

Anhand der Intraklassenkorrelationen der Baseline-Modelle zeigte sich auf allen Affiliationsperspektiven, dass ca. die Hälfte der Varianz an Affiliation durch interindividuelle Unterschiede erklärt wurde (Affiliation des eigenen therapeutischen Handelns: $51 \%$; Affiliation des Verhaltens der Klient:innen: $51 \%$,Affiliation in Bezug auf sich selbst: $43 \%$ ).

Die wahrgenommene Affiliation des eigenen therapeutischen Handelns ver- besserte sich im Mittel signifikant über die Zeit mit einer kleinen Effektstärke ( $B=8,19$; 95\%-Konfidenzintervall [95\%KI] 2,91-13,88; $p=0,005 ; d=0,26$ ). Die Affiliationswerte dieser Perspektive nach 3 Jahren wurden signifikant durch Bindungsvermeidung zu Beginn der Ausbildung vorhergesagt $(B=-17,42 ; 95 \%-K I$ $-30,21$ bis $-4,64 ; p=0,007$ ), wobei höhere Ausprägungen mit geringerer Affiliation assoziiert waren (• Abb. 1). Bindungsangst hatte in diesem Model keinen signifikanten prädiktiven Wert $(B=2,15 ; 95 \%-K I$ $-6,80-11,10 ; p=0,64)$.

Die wahrgenommene Affiliation des Verhaltens der Klient:innen zeigte im
Mittel ebenfalls eine signifikante Zunahme über die Zeit mit einer kleinen Effektstärke ( $B=8,10 ; 95 \%-K I$ 1,69-14,52; $p=0,01 ; d=0,23)$. Zudem fanden sich signifikante Interaktionen zwischen den Prädiktoren Zeit und Selbsterfahrungsstunden $(B=-0,12 ; 95 \%-\mathrm{KI}-0,05$ bis-3,43; $p<0,001)$, wobei AK mit weniger Selbsterfahrungsstunden ihre Klient:innen über die Zeit als zugewandter wahrnahmen (• Abb. 2). Des Weiteren zeigte sich eine signifikante Interaktion zwischen Bindungsvermeidung und Selbsterfahrungsstunden $(B=0,17 ; 95 \%-K I \quad 0,01$, 0,$32 ; p=0,03$ ), bei der AK mit hoher Bindungsvermeidung und vielen Selbst- 


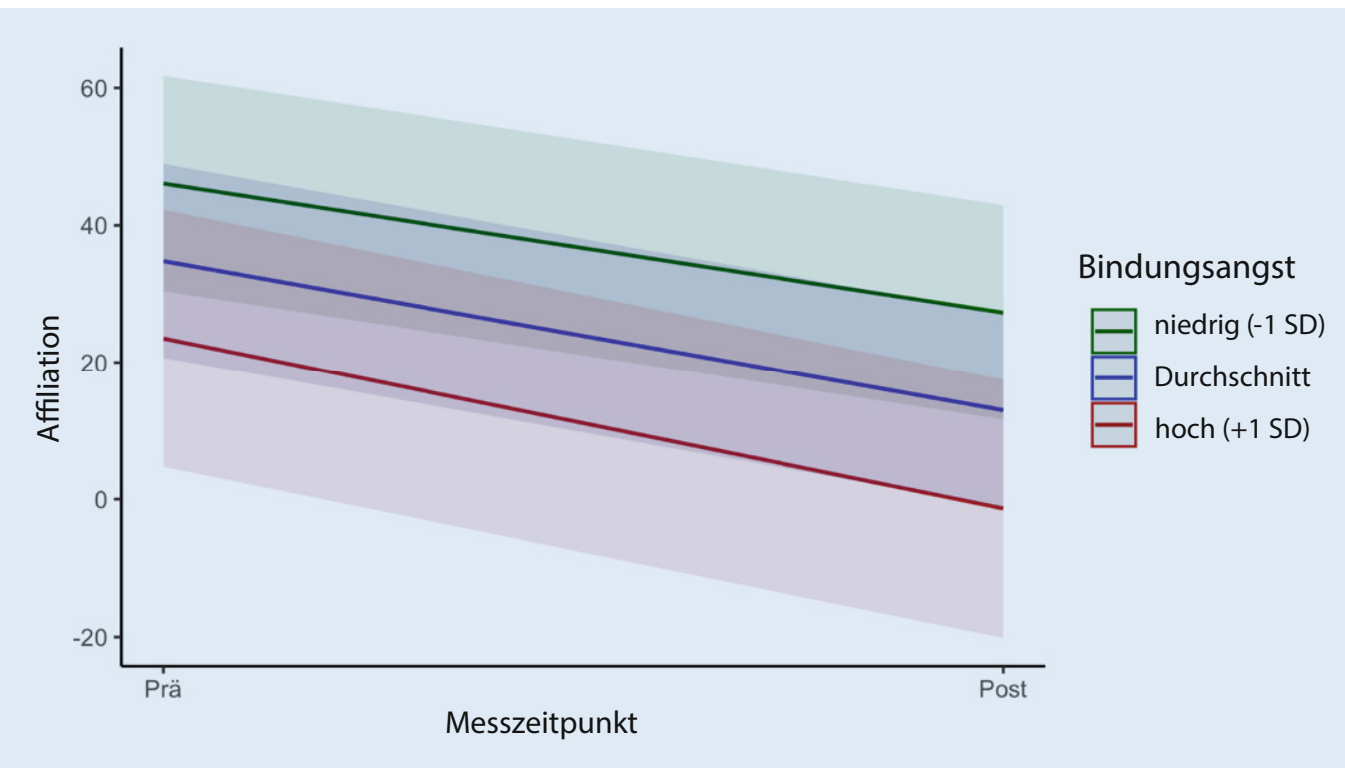

Abb. 34 Entwicklung der selbstbezogenen Affiliation in Abhängigkeit von Bindungsangst

erfahrungsstunden ihre Klient:innen als affiliativer wahrnahmen. Bindungsangst hatte keinen signifikanten prädiktiven Wert in diesem Model $(B=0,57 ; 95 \%-K I$ $-9,56-10,71 ; p=0,91)$.

Die wahrgenommene Affiliation der $A K$ in Bezug auf sich selbst zeigte eine signifikante Verschlechterung von einer mittleren Effektstärke über 3 Jahre $(\mathrm{B}=-21,93 ; 95 \%-\mathrm{Kl}-30,12$ bis $-13,73$; $p<0,001, d=0,48)$. Hohe Ausprägungen auf Bindungsangst zu Beginn der Ausbildung sagten eine geringere selbstbezogene Affiliation zu T2 signifikant voraus $(B=-11,85 ; 95 \%-K I \quad-22,56$ bis $-1,14$; $p=0,03$; Abb. 3). Bindungsvermeidung erreichte in diesem Modell kein signifikantes Regressionsgewicht $(B=-8,93 ; 95 \%$ $\mathrm{KI}-24,24-6,38 ; p=0,25$ ).

Die Zufriedenheit mit Selbsterfahrung hatte über alle Perspektiven keinen zusätzlichen prädiktiven Wert. Die detaillierten Parameterschätzungen aller 3 Multilevelmodelle zeigt $\bullet$ Tab. 2 .

\section{Diskussion}

In der vorliegenden Stichprobe von AK fanden sich eine Zunahme der interpersonellen Affiliation mit einer kleinen Effektstärke über 3 Jahre sowie eine Abnahme intrapsychischer Affiliation mit einer mittleren Effektstärke. Die AK wiesen damit nach 3 Jahren Ausbildung eine wohlwollendere Haltung ihren Klient:innen gegenüber und eine weniger wohlwollende $\mathrm{Hal}$ - tung sich selbst gegenüber auf. Etwa die Hälfte der Varianz in allen Affiliationsbereichen wurde durch interindividuelle Unterschiede erklärt. Hohe Ausprägungen an Bindungsvermeidung zu Beginn der Ausbildung sagten eine geringere Affiliation im eigenen therapeutischen Verhalten voraus. Hohe Ausprägungen an Bindungsangst gingen mit einer geringeren Affiliation in Bezug auf sich selbst nach 3 Jahren einher. Die Zufriedenheit mit der Selbsterfahrung hatte keinen prädiktiven Wert. Die Länge der Selbsterfahrung zeigte einzig einen geringfügigen negativen Einfluss auf die wahrgenommene Affiliation im Verhalten der Klient:innen, wobei sich dieser Effekt bei stärker bindungsvermeidenden AK umkehrte.

\section{Interpretation der Ergebnisse}

Der Befund, dass AK ihr eigenes therapeutisches Verhalten als wohlwollender und das Verhalten der Klient:innen als weniger feindselig wahrnehmen, spricht dafür, dass es innen im Verlauf der Ausbildung gelingt, gelassener mit schwierigen Situationen umzugehen und mehr Empathie für den Störungsausdruck ihrer Klient:innen aufzubringen. Die gleichzeitig vorliegende deutliche Abnahme von selbstbezogener Affiliation bedeutet, dass AK im Ausbildungsverlauf weniger wohlwollend mit sich selbst umgehen, und steht im Widerspruch zu vorausgegangener Affiliationsforschung (Taubner et al. 2013). Den- noch könnten die Ergebnisse gemäß dem Phasenmodell zur Entwicklung von Psychotherapeut:innen von Rønnestad und Skovholt (2013) einen normativen Befund darstellen. Das Modell geht davon aus, dass AK nach einigen Jahren Ausbildung zwar ein Basisniveau an therapeutischem Funktionieren etablieren, jedoch häufig unrealistisch hohe, perfektionistische Standards an das eigene Arbeiten stellen. Bei einer empirischen Untersuchung des Modells fand sich, dass AK in fortgeschrittener Ausbildung unter Diskrepanzen zwischen ihrem Real- und Idealselbst in Bezug auf die eigene innere Abgrenzung und emotionale Vulnerabilität leiden (Tilkidzhieva et al. 2019). Ein solcher Vergleich der eigenen Leistung mit hohen Ich-Idealen in Bezug auf das therapeutische Vorgehen könnte beispielsweise in Selbstabwertungen münden, die sich hier empirisch als Abnahme der selbstbezogenen Affiliation darstellen. Die Abnahme könnte ebenfalls durch Faktoren außerhalb des Klient:innenkontakts beeinflusst worden sein (Taubner et al. 2013), beispielsweise durch die allgemein hohe Ausbildungsbelastung (Grundmann et al. 2013) oder Belastungen im Privatleben der AK. In jedem Fall sollte dieser Entwicklung entgegengewirkt werden, da eine selbstabwertende Haltung gegen Ende der Therapieausbildung das Risiko für Belastungen im Klient:innenkontakt und eine negative professionelle Entwicklung erhöhen kann. 
Tab. 2 Mehrebenenmodelle zur Vorhersage der Affiliationswerte

\begin{tabular}{|c|c|c|c|c|c|c|c|c|c|}
\hline \multirow[b]{2}{*}{ Prädiktoren } & \multicolumn{3}{|c|}{ Selbstbezogene Affiliation } & \multicolumn{3}{|c|}{ Affiliation therapeutisches Verhalten } & \multicolumn{3}{|c|}{ Affiliation Klient:innenverhalten } \\
\hline & B & $S E$ & $p$ & B & $S E$ & $p$ & B & $S E$ & $p$ \\
\hline Intercept & 36,18 & 7,74 & $<0,001$ & 19,42 & 6,76 & 0,004 & $-27,78$ & 7,67 & $<0,001$ \\
\hline Alter & 1,77 & 0,58 & 0,002 & 0,88 & 0,51 & 0,09 & 1,31 & 0,58 & 0,02 \\
\hline Geschlecht & $-17,16$ & 9,65 & 0,08 & $-5,03$ & 8,55 & 0,56 & $-2,47$ & 9,68 & 0,80 \\
\hline Semester & 1,44 & 2,15 & 0,50 & $-0,01$ & 1,90 & 0,99 & $-2,76$ & 2,16 & 0,20 \\
\hline VT vs. PD & $-14,78$ & 8,33 & 0,08 & 8,51 & 7,38 & 0,25 & 6,64 & 8,36 & 0,43 \\
\hline Zeit & $-21,93$ & 4,18 & $<0,001$ & 8,19 & 2,91 & 0,005 & 8,10 & 3,27 & 0,01 \\
\hline Bindungsangst & $-11,85$ & 5,46 & 0,03 & 2,15 & 4,57 & 0,64 & 0,57 & 5,17 & 0,91 \\
\hline Bindungsvermeidung & $-8,93$ & 7,81 & 0,25 & $-17,42$ & 6,52 & 0,007 & $-7,98$ & 7,38 & 0,28 \\
\hline SE, Zufriedenheit & 9,16 & 4,92 & 0,06 & 2,46 & 4,15 & 0,55 & $-5,12$ & 4,70 & 0,28 \\
\hline SE, Stunden & 0,03 & 0,05 & 0,59 & $-0,01$ & 0,04 & 0,88 & 0,04 & 0,04 & 0,35 \\
\hline Zeit•BA & $-3,24$ & 5,83 & 0,58 & $-5,10$ & 4,05 & 0,21 & $-1,27$ & 4,56 & 0,78 \\
\hline Zeit• BV & 3,02 & 8,41 & 0,72 & 10,63 & 5,85 & 0,07 & 2,04 & 6,59 & 0,76 \\
\hline Zeit• Zuf & $-1,21$ & 4,86 & 0,80 & $-1,73$ & 3,38 & 0,61 & 6,12 & 3,80 & 0,11 \\
\hline Zeit• Std & $-0,03$ & 0,05 & 0,59 & $-0,04$ & 0,03 & 0,25 & $-0,12$ & 0,04 & 0,001 \\
\hline BA•Zuf & $-5,35$ & 5,03 & 0,29 & $-2,74$ & 4,46 & 0,54 & $-0,85$ & 5,05 & 0,87 \\
\hline $\mathrm{BA} \bullet \mathrm{Std}$ & $-0,03$ & 0,05 & 0,52 & $-0,06$ & 0,05 & 0,21 & $-0,10$ & 0,05 & 0,07 \\
\hline BV• Zuf & $-1,40$ & 7,94 & 0,86 & 3,02 & 7,03 & 0,67 & 1,07 & 8,00 & 0,90 \\
\hline $\mathrm{BV} \bullet \mathrm{Std}$ & $-0,06$ & 0,08 & 0,42 & 0,08 & 0,07 & 0,26 & 0,17 & 0,08 & 0,03 \\
\hline
\end{tabular}

$B A$ Bindungsangst, $B V$ Bindungsvermeidung, VT vs. $P D$ verhaltenstherapeutische Ausbildung vs. psychodynamische Ausbildung, SE Selbsterfahrung, Std kumulierte Stunden Selbsterfahrung zur Postmessung, Zuf Zufriedenheit mit der Selbsterfahrung

Ein bedeutsamer Teil der inter- und intrapersonellen Affiliation wurde durch interindividuelle Unterschiede erklärt. Dabei spricht der signifikante Einfluss der Bindungsrepräsentationen für die Annahme, dass das therapeutische Erleben in schwierigen Situationen z.T. durch verinnerlichte zwischenmenschliche Schemata der AK geprägt wird. Die gefundene niedrigere Affiliation im eigenen therapeutischen Verhalten von AK mit hoher Bindungsvermeidung ist theoretisch dadurch erklärbar, dass diese in emotional fordernden Situationen eher mit emotionsdeaktivierenden Strategien reagieren (Dykas und Cassidy 2011). Demzufolge könnten sie sich in ihren Interventionen möglicherweise stärker auf sachliche Inhalte fokussieren und das gemeinsame Explorieren und Aushalten von Emotionen meiden. Der negative Einfluss von Bindungsangst auf die selbstbezogene Affiliation legt den Schluss nahe, dass hochbindungsängstliche AK aus Angst vor Zurückweisung (Mikulincer und Shaver 2007) eine situative Überforderung eher auf die eigene Unzulänglichkeit attribuieren, um die Beziehung zu ihrem Gegenüber zu schützen. Entgegen den Annahmen hatte keine Bindungsdimension direkten Einfluss auf die Wahrnehmung der Affiliation des Klient:innenverhaltens. Das bedeutet, dass sich unsichere Bindungsrepräsentationen in dieser Stichprobe, entgegen den theoretisch damit assoziierten negativen Schemata (Mikulincer und Shaver 2007), nicht auf die Wahrnehmung des Gegenübers auswirkten. Zusammengenommen deuten diese Befunde darauf hin, dass interpersonelle Erfahrungen die Affiliation der AK im Sinne eines Trait prägen, die Affiliation aber für plastische Entwicklungsprozesse während der Ausbildung zugänglich bleibt.

Ebenfalls überraschte, dass Selbsterfahrungsmaße nur wenig Einfluss auf die Affiliationsentwicklung nahmen. Dieser Befund könnte dadurch zu erklären sein, dass in qualitativen Studien zur Selbsterfahrung neben positiven Effekten gleichermaßen unerwünschte negative Effekte beschrieben werden (z.B. emotionale Belastung und Destabilisierung bestehender Beziehungen; Murphy et al. 2018). Diese Effekte könnten sich im Mittel gegenseitig aufheben und sich empirisch im vorliegenden Befund widerspiegeln. Einzig die wahrgenommene Affiliation im Klient:innenverhalten wurde durch die Dauer der Selbsterfahrung geprägt, wobei zwei divergierende Befunde auf eine dif- ferenzielle Wirkung von Selbsterfahrung hinweisen. Während eine höhere Zahl von Selbsterfahrungsstunden generell mit einer geringeren wahrgenommenen Affiliation assoziiert war, kehrte sich dieser Effekt bei AK mit einer höheren Bindungsvermeidung um. Durch das naturalistische Forschungsdesign könnten verschiedene inhaltliche Faktoren in diesen Effekten konfundiert sein. Beispielsweise könnten interpersonelle Schwierigkeiten im Klient:innenkontakt generell dazu geführt haben, dass AK mehr Selbsterfahrung in Anspruch nahmen. Bei stark bindungsvermeidenden AK könnte die Wahrnehmung des Gegenübers wiederum in der Selbsterfahrung stärker fokussiert und dadurch eine zugewandtere Haltung gefördert worden sein.

Da weder das Ausmaß von noch die Zufriedenheit mit der Selbsterfahrung einen positiven Einfluss auf die Entwicklung einer wohlwollenden Haltung im eigenen therapeutischen Handeln und der Selbstwahrnehmung hatten, sollten zukünftige Studien klären, welche Arten von Selbsterfahrung in diesem Kontext förderlich und ressourcenstärkend sind. 


\section{Limitationen}

Methodische Limitationen betreffen v.a. die Operationalisierung der erfassten Konstrukte. Bei der Erhebung von Affiliation durch den Intrex-Fragebogen wurden AK zu einer Einschätzung ihres Verhaltens in schwierigen Situationen über alle Klient:innen hinweg aufgefordert. Dadurch sind ein Informationsverlust zu einzelnen Interaktionen sowie eine Anfälligkeit für soziale Erwünschtheit, Heuristiken und Erinnerungseffekte anzunehmen. Zudem wurde die Entwicklung von Affiliation in sehr guten Therapiesitzungen (,zur besten Zeit") nicht untersucht, da theoriegeleitet ein stärkerer Einfluss von Bindungsrepräsentationen auf schwierige Sitzungen („zur schlechtesten Zeit") erwartet wurde. Die Beschränkung auf Dauer und Zufriedenheitsmaße der Selbsterfahrung lässt wenige inhaltliche Schlüsse über mögliche Prozessmaße zu. Ferner wurden Einflüsse aus dem Privatleben der AK und deren bisherige Berufserfahrung nicht als mögliche Prädiktoren erfasst. Auch wenn die Bindungsmaße im Mittel keine Veränderung über die Ausbildung zeigten, ist deren statische Behandlung in Anbetracht der theoretisch engen Verknüpfung von Affiliation und Bindungsrepräsentationen ebenfalls disputabel (Gallo et al. 2003). In der Stichprobe überwogen prozentual AK in psychodynamischen Verfahren, was die Generalisierbarkeit auf die Gesamtheit deutscher AK mit hohem verhaltenstherapeutischem Anteil einschränkt. Zudem fand sich für die längsschnittlichen Kriterien ein substanzieller Drop-out von $32 \%$. Die vorliegenden Ergebnisse einer "Complete-cases"-Analyse bergen potenziell eine Überschätzung positiver und eine Unterschätzung negativer Effekte (Jakobsen et al. 2017).

\section{Fazit für die Praxis}

- Im Verlauf ihrer Ausbildung entwickeln Ausbildungskandidat:innen eine wohlwollendere Wahrnehmung ihres eigenen therapeutischen Handelns und des Verhaltens ihrer Klient:innen.

- Ausbildungskandidat:innen neigen im Verlauf der Ausbildung zu einer zunehmend selbstabwertenden Haltung. Dem sollte durch eine stärkere Betonung von Ressourcen und Selbstfürsorge in der Ausbildung begegnet werden.
- Bindungsangst und Bindungsvermeidung sagen eine weniger wohlwollende $\mathrm{Hal}-$ tung gegenüber Klient:innen und sich selbst vorher. Daher sollten diese in der Ausbildung ausreichend berücksichtigt werden.

\section{Korrespondenzadresse}

\section{Eric Thanbichler}

Institut für Psychosoziale Prävention, Zentrum für Psychosoziale Medizin, Universitätsklinikum Heidelberg

Bergheimer Str. 54, 69115 Heidelberg, Deutschland

eric.thanbichler@med.uni-heidelberg.de

Förderung. Gefördert von der Deutschen Forschungsgemeinschaft (MO 2008/2-1, MO 2008/22).

Funding. Open Access funding enabled and organized by Projekt DEAL.

\section{Einhaltung ethischer Richtlinien}

Interessenkonflikt. E. Thanbichler, O. Evers, H. Möller und S. Taubner geben an, dass kein Interessenkonflikt besteht.

Die Teilnahme an der Studie erfolgte anonymisiert und freiwillig. Die ethische Unbedenklichkeit dieser Studie wurde von der Ethikkomission der Universität Kassel bestätigt. Von allen Teilnehmer:innen liegt eine Einverständniserklärung vor.

Open Access. Dieser Artikel wird unter der Creative Commons Namensnennung 4.0 International Lizenz veröffentlicht, welche die Nutzung, Vervielfältigung, Bearbeitung, Verbreitung und Wiedergabe in jeglichem Medium und Format erlaubt, sofern Sie den/die ursprünglichen Autor(en) und die Quelle ordnungsgemäßnennen, einen Link zur Creative Commons Lizenz beifügen und angeben, ob Änderungen vorgenommen wurden.

Die in diesem Artikel enthaltenen Bilder und sonstiges Drittmaterial unterliegen ebenfalls der genannten Creative Commons Lizenz, sofern sich aus der Abbildungslegende nichts anderes ergibt. Sofern das betreffende Material nicht unter der genannten Creative Commons Lizenz steht und die betreffende Handlung nicht nach gesetzlichen Vorschriften erlaubt ist, ist für die oben aufgeführten Weiterverwendungen des Materials die Einwilligung des jeweiligen Rechteinhabers einzuholen.

Weitere Details zur Lizenz entnehmen Sie bitte der Lizenzinformation auf http://creativecommons.org/ licenses/by/4.0/deed.de.

\section{Literatur}

Benjamin LS (1974) Structural analysis of social behavior. Psychol Rev 81(5):392-425. https:// doi.org/10.1037/h0037024

Benjamin LS (1995) Intrex user's manual. University of Utah,

BowlbyJ (1988) A secure base: parent-childattachment and healthy human development. Basic Books,

Bruck E, Winston A, Aderholt S, Muran JC (2006) Predictive validity of patient and therapist attachment and introject styles. Am J Psychother 60(4):393-406. https://doi.org/10.1176/appi. psychotherapy.2006.60.4.393

Deal KH, Bennett S, Mohr J, Hwang J (2011) Effects of field instructor training on student competencies and the supervisory alliance. Res Soc Work Pract 21(6):712-726. https://doi.org/ 10.1177/1049731511410577

Dykas MJ, Cassidy J (2011) Attachment and the processing of social information across the life span: theory and evidence. Psychol Bull 137(1):19-46. https://doi.org/10.1037/a0021367

Ehrenthal J, Dinger U, Lamla A, Funken B, Schauenburg $\mathrm{H}$ (2009) Evaluation der deutschsprachigen Version des Bindungsfragebogens "Experiences in Close Relationships-Revised" (ECRRD). Psychother Psychosom Med Psychol 59(06):215-223. https://doi.org/10.1055/s2008-1067425

Gallo LC, Smith TW, Ruiz JM (2003) An interpersonal analysis of adult attachment style: circumplex descriptions, recalled developmental experiences, self-representations, and interpersonal functioning in adulthood: an interpersonal analysis of adult attachment. J Pers 71(2):141-182. https://doi.org/10.1111/1467-6494.7102003

Gold SH, Hilsenroth MJ (2009) Effects of graduate clinicians' personal therapy on therapeutic alliance. Clin Psychol Psychother 16(3):159-171. https://doi.org/10.1002/cpp.612

Grundmann J, Sude K, Löwe B, Wingenfeld K (2013) Arbeitsbezogene Stressbelastung und psychische Gesundheit: Eine Befragung von Psychotherapeutinnen und -therapeuten in Ausbildung [Work-related Behaviour and Experience Patterns and Mental Health: a Study in Psychotherapy Trainees]. Psychother Psychosom Med Psychol 63(03/04):145-149. https://doi. org/10.1055/s-0032-1333292

Heinonen E, Nissen-Lie HA (2020) The professional and personal characteristics of effective psychotherapists: a systematic review. Psychother Res. https://doi.org/10.1080/10503307.2019. 1620366

Henry WP, Schacht TE, Strupp HH (1990) Patient and therapist introject, interpersonal process, and differential psychotherapy outcome. J Consult Clin Psychol 58(6):768-774. https://doi.org/10. 1037//0022-006X.58.6.768

Jakobsen JC, Gluud C, Wetterslev J, Winkel P (2017) When and how should multiple imputation be used for handling missing data in randomised clinical trials-a practical guide with flowcharts. BMC Med Res Methodol 17(1):162. https://doi. org/10.1186/s12874-017-0442-1

Johns RG, Barkham M, Kellett S, Saxon D (2019) A systematic review of therapist effects: a critical narrative update and refinement to Baldwin and Imel's (2013) review. Clin Psychol Rev 67:78-93. https://doi.org/10.1016/j.cpr.2018.08.004

Mikulincer M, Shaver PR (2007) Attachment in adulthood: structure, dynamics, and change. Guilford, 
Mikulincer M, Gillath O, Shaver PR (2002) Activation of the attachment system in adulthood: threatrelated primes increase the accessibility of mental representations of attachment figures. J Pers Soc Psychol 83(4):881-895. https://doi. org/10.1037/0022-3514.83.4.881

Mohr JJ, Gelso CJ, Hill CE (2005) Client and counselor trainee attachment as predictors of session evaluation and countertransference behavior in first counseling sessions. J Couns Psychol 52(3):298-309. https://doi.org/10.1037/00220167.52.3.298

Murphy D, Irfan N, Barnett $\mathrm{H}$, Castledine $\mathrm{E}$, Enescu $L$ (2018) A systematic review and metasynthesis of qualitative research into mandatory personal psychotherapy during training. Couns Psychother Res 18(2):199-214. https://doi.org/ 10.1002/capr.12162

Nienhuis JB, Owen J, Valentine JC, Winkeljohn Black S, Halford TC, Parazak SE, Budge S, Hilsenroth $M$ (2018) Therapeutic alliance, empathy, and genuineness in individual adult psychotherapy: a meta-analytic review. Psychother Res 28(4):593-605. https://doi.org/10.1080/ 10503307.2016.1204023

Nissen-Lie HA, Rønnestad MH, Høglend PA, Havik OE, Solbakken OA, Stiles TC, Monsen JT (2017) Love yourself as a person, doubt yourself as a therapist?: Relationship of therapist professional and personal functioning to therapeutic outcome. Clin Psychol Psychother 24(1):48-60. https://doi.org/10.1002/cpp.1977

Pincus AL, Dickinson KA, Schut AJ, Castonguay LG, Bedics J (1999) Integrating Interpersonal Assessment and Adult Attachment Using SASB. Eur J Psychol Assess 15(3):206-220. https://doi. org/10.1027//1015-5759.15.3.206

Pincus AL, Newes SL, Dickinson KA, Ruiz MA (1998) A comparison of three indexes to assess the dimensions of structural analysis of social behavior. J Pers Assess 70(1):145-170. https:// doi.org/10.1207/s15327752jpa7001_10

Rønnestad MH, Skovholt TM (2013) The developing practitioner: growth and stagnation of therapists and counselors. Routledge, Taylor \& Francis Group,

Rubino G, Barker C, Roth T, Fearon P (2000) Therapist empathy and depth of interpretation in response topotentialallianceruptures:therole of therapist and patient attachment styles. Psychother Res 10(4):408-420. https://doi.org/10.1093/ptr/10. 4.408

Sandell R, Taubner S, Rapp A, Visbeck A, Kächele H (2008) Psycho-Therapeutische Haltung Ausbildungsversion (ThAt-AV). Universität UIm, Ulm

Schauenburg $\mathrm{H}$, Buchheim A, Beckh K, Nolte T, BrenkFranz K, Leichsenring F, Strack M, Dinger U (2010) The influence of psychodynamically oriented therapists' attachment representations on outcome and alliance in inpatient psychotherapy. Psychother Res 20(2):193-202. https://doi.org/ 10.1080/10503300903204043

Schut AJ, Castonguay LG, Flanagan KM, Yamasaki AS, Barber JP, Bedics JD, Smith TL (2005) Therapist interpretation, patient-therapist interpersonal process, and outcome in psychodynamic psychotherapy for avoidant personality disorder. Psychotherapy 42(4):494-511. https://doi.org/ 10.1037/0033-3204.42.4.494

Shaver PR, Collins N, Clark CL (1996) Attachment styles and internal working models of self and relationship partners. In: Knowledge structures

\section{Development of affiliation during psychotherapy training. Towards a benevolent attitude}

Objective: The study investigated the development of affiliation in psychotherapy trainees with respect to their therapeutic conduct, their self-perception and their perception of clients. In addition, the influence of attachment representations and personal therapy on affiliation was examined.

Material and methods: In a naturalistic pre-post design, trainees of different therapeutic orientations $(n=126)$ rated different dimensions of affiliation in difficult therapy situations (Intrex questionnaire short form) over an interval of 3 years. Attachment anxiety and attachment avoidance were assessed at baseline (experiences in close relationships, ECR-RD) as well as length of and satisfaction with self-experience at the end of training. Hierarchical linear modeling was implemented for data analyses. Results: Affiliation of the trainees' behavior towards clients and their perception of client behavior showed increases of a small effect size, whereby attachment avoidance predicted a lower affiliation in own behavior. Self-related affiliation showed a decrease with a medium effect size and attachment anxiety was associated with a lower affiliation. The duration of self-experience showed a negative association with the perception of client behavior, although this effect was reversed with high levels of attachment avoidance. The satisfaction with self-experience had no influence on the development of affiliation.

Conclusion: The interpersonal and intrapsychic affiliation of trainee psychotherapists is partly influenced by attachment representations but is subject to developmental processes. The decrease in self-related affiliation shows the necessity of compensatory and resource strengthening measures in psychotherapy training.

\section{Keywords}

Attachment representation - Interpersonal perception - Self-perception - Personal therapy . Therapist effects

in close relationships: a social psychological approach. Lawrence Erlbaum, , S25-61

Slade A, Holmes J (2019) Attachment and psychotherapy. Curr Opin Psychol 25:152-156. https://doi. org/10.1016/j.copsyc.2018.06.008

Sroufe LA, Egeland B, Carlson EA, Collins WA (2005) The development of the person: the Minnesota study of risk and adaptation from birth to adulthood. Guilford,

Tabachnick BG, Fidell LS (2014) Using multivariate statistics. Pearson,

Taubner S, Zimmermann J, Kächele H, Möller H, Sell C (2013) The relationship of introject affiliation and personal therapy to trainee self-efficacy: a longitudinal study among psychotherapy trainees. Psychotherapy 50(2):167-177. https:// doi.org/10.1037/a0029819

Taubner S, Klasen J, Hanke W, Möller H (2015) Ein empirischer Zugang zur Erfassung der Kompetenzentwicklung von PsychotherapeutInnen in Ausbildung. Psychother Forum 20(1-2):47-53. https://doi.org/10.1007/s00729-015-0035-6

Tilkidzhieva E, Gelo OCG, Gullo S, Orlinsky DE, Mörtl K, Fiegl J (2019) Self-concept of relational skills in psychotherapy trainees: a pilot study. Couns Psychother Res 19(3):311-320. https://doi.org/ 10.1002/capr.12217 